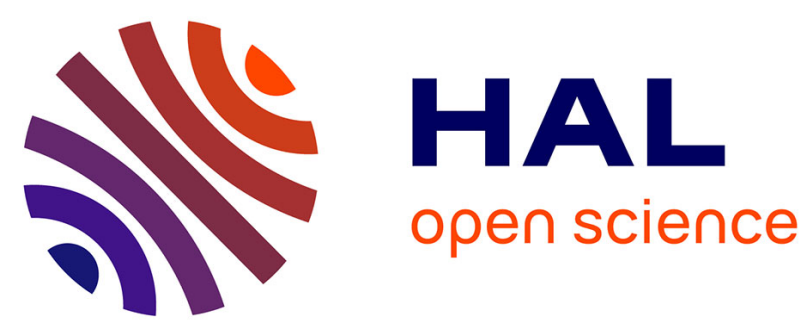

\title{
L'évolution de la descendance finale en France des générations 1928-1939
}

Gérard-François Dumont, P Descroix

\section{To cite this version:}

Gérard-François Dumont, P Descroix. L'évolution de la descendance finale en France des générations 1928-1939. Population (édition française), 1991, 46 (5), pp.1268-1271. 10.2307/1533463 . halshs02558418

\section{HAL Id: halshs-02558418 https://shs.hal.science/halshs-02558418}

Submitted on 29 Apr 2020

HAL is a multi-disciplinary open access archive for the deposit and dissemination of scientific research documents, whether they are published or not. The documents may come from teaching and research institutions in France or abroad, or from public or private research centers.
L'archive ouverte pluridisciplinaire HAL, est destinée au dépôt et à la diffusion de documents scientifiques de niveau recherche, publiés ou non, émanant des établissements d'enseignement et de recherche français ou étrangers, des laboratoires publics ou privés. 


\section{L'évolution de la descendance finale en France des générations} 1928-1939

G. F. Dumont, P. Descroix

Citer ce document / Cite this document :

Dumont G. F., Descroix P. L'évolution de la descendance finale en France des générations 1928-1939. In: Population, 46 année, $n^{\circ} 5,1991$. pp. 1268-1271;

doi : $10.2307 / 1533463$

https://www.persee.fr/doc/pop_0032-4663_1991_num_46_5_3744

Fichier pdf généré le 26/04/2018 


\section{L'ÉVOLUTION DE LA DESCENDANCE FINALE EN FRANCE DES GÉNÉRATIONS 1928-1939}

Dans une précédente étude, nous avons examiné l'évolution de la fécondité des femmes en France et souligné la régularité non négligeable de cette évolution de 1963 à $1978^{(1)}$. Il s'agissait là, selon la distinction classique, d'un regard sur l'évolution transversale de la fécondité. L'analyse longitudinale, quant à elle, a l'inconvénient d'avoir «un caractère largement rétrospectif » et «des difficultés de reconstructions "(2). Ce dernier inconvénient, en ce qui concerne les donnécs relatives à la descendance finale des femmes résidant en France métropolitaine, n'existe pas puisque l'INSEE livre des données permettant d'analyser cette évolution(3).

Plusieurs caractéristiques se dégagent :

1) La descendance finale des femmes ayant atteint l'âge de 50 ans de 1978 à 1989 (générations nées entre 1928 et 1939) apparaît supérieure au niveau du simple remplacement des générations qui, pour la période de vie féconde de ces femmes, peut être estiméc entre 2,20 et 2,10 enfants par femme. Elle est largement supérieure à l'indice synthétique de fécondité des douze dernières années, ce qui signifie que le comportement de fécondité des femmes actuellement en âge de procréer est inféricur au comportement des générations considérées.

2) Le maximum de la descendance finale a été de 263,4 pour 100 femmes nées en 1930. Il est ensuite en baisse régulic̀re.

3) La connaissance des chiffres des générations 1928-1939 permet de définir, grâce à un logiciel approprić, une équation du second degré :

$$
Y=262,458+0,869 X-0,201 X^{2}
$$

où $Y$ est la descendance finale des femmes nées l'année $X$ (avec $1928=1$ et $1939=12$ ). Cette équation du second degré est visualisée par unc courbe sur le graphique 1 .

4) Cette courbe de régression du second degré est d'une grande régularité. Cette régularité peut se comprendre du fait que, statistiquement, la descendance finale d'une femme est le cumul des taux enregistrés au cours des trente cinq années de sa vie fertile, qui se reflètent, annéc après année, même s'il n'existe pas de corrélation mathématique absolue, dans l'indice synthétique de fécondité.

Or cet indice a enregistré en France des variations relativement faibles d'une annéc à l'autre. Pendant la période où ses variations ont été les plus rapides, de 1964 (ISF $=290$ enfants pour 100 femmes) à $1976($ ISF $=193)$ cette variation n'a été en moyenne que de $8 \%$ par an. De plus, l'indice synthétique est resté pratiquement stable pendant les douze années suivantes, de $1977(=186)$ à $1989(=181)$. Cette logique, si elle se vérifiait, autorise à extrapoler la courbe de régression ci-

(1) Gérard-François Dumont et Pierre Descroix, «La fécondité de la France par rapport à celle des pays industriels », Population, XXXXIV, 2, 1989, p. 441-444.

(2) Roland Pressat, Dictionnaire de la démographie, Paris, PUF, 1979, p. 105.

(3) La situation démographique en 1988, INSEE Résultats, série Démographie-Société, no 3-4, juillet 1990 , p. 94 . Les chiffres de descendance finale des générations 1928 et 1939 nous ont été aimablement communiqués oralement par Yves Court, leur publication étant prévue courant 1991. 
dessous. Dans ce cas, une descendance finale de 2,1 , niveau au-dessous duquel le remplacement des générations n'est plus assuré, serait atteinte, par la génération née en 1946.

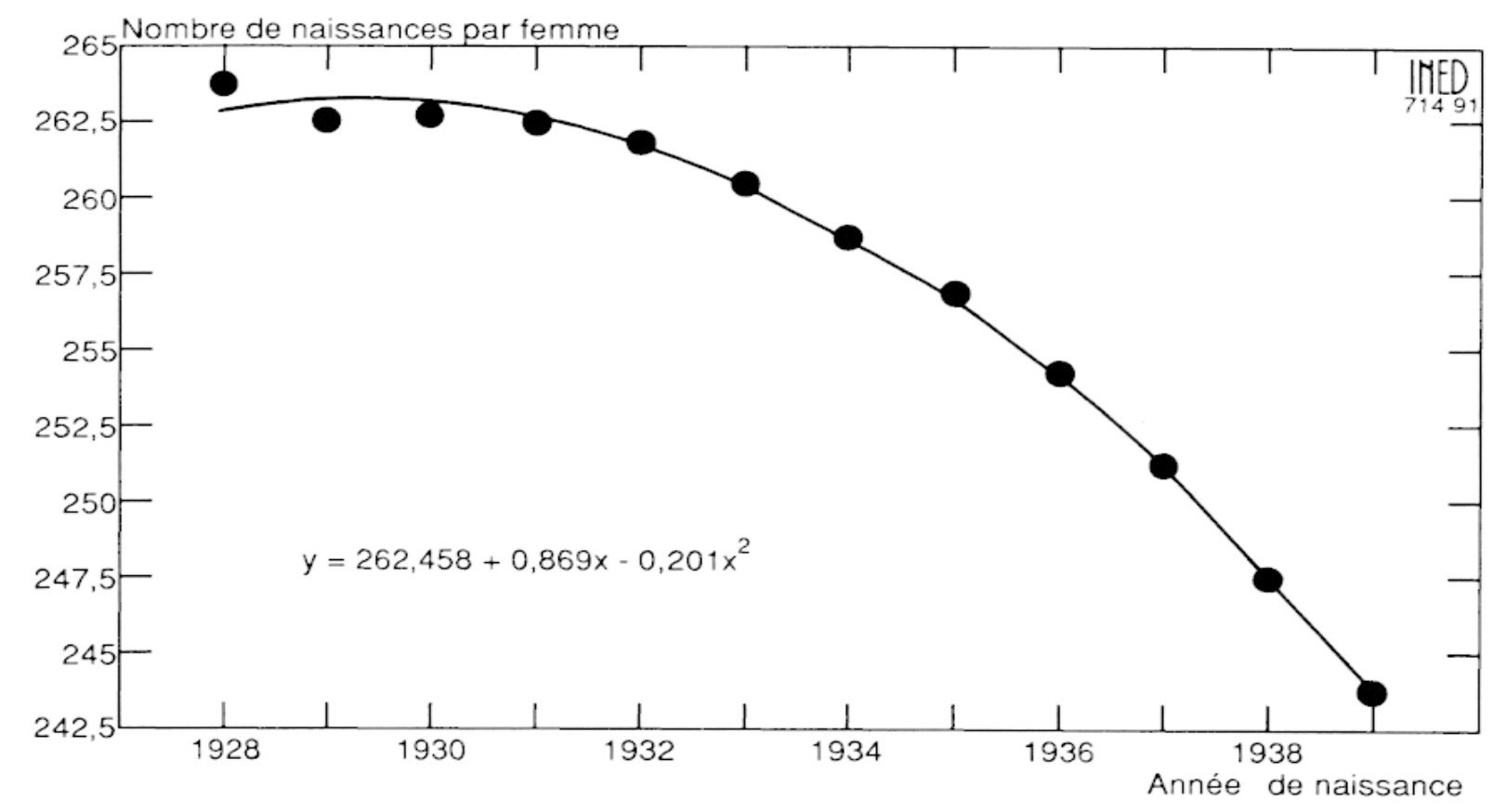

\section{Graphique 1. - La descendance finale des femmes en France pour 100 femmes (générations $1928=1$ à $1939=12$ )}

Il est difficile de prévoir quand cette parabole s'infléchira pour laisser place à une courbe d'allure anti-logistique, suite normale de ce genre d'évolution(4), qui devrait tendre vers une asymptote d'environ 180 , valeur autour de laquelle s'est stabilisé l'indice synthétique de fécondité en France depuis 1975. Il n'y a pour l'instant aucun signe d'un tel infléchissement.

Si l'on trace en effet les courbes de régression pour les générations 19281937, 1928-1938, 1928-1939, en calculant ensuite par extrapolation la valeur qui serait atteinte dans la génération 1945 ( $18^{\text {ṁme }}$ année), on trouve successivement 216,02 , puis 214,25 et enfin 212,98 , c'est-à-dire que la diminution du nombre d'enfants que compteront les femmes lorsque les générations intéressées atteindront cinquante ans, n’a fait que s'accélérer dans les trois dernières générations.

5) Pour affiner celte étude, rappelons que l'INSEE donne également, dans le document cité ci-dessus, la descendance (non finale) des femmes n'ayant pas encore terminé leur période fertile. Les chiffres correspondants permettent de porter les observations suivantes, en regroupant ces données en trois intervalles : 14 à 24 ans, 25 à 35 ans, 36 à 50 ans.

(4) Marc Artzouni. "Une nouvelle famille de courbes de croissance. Application à la transition démographique », Population, XXXX1, 3, 1986, p. 497-508. 
Ces intervalles correspondent approximativement aux trois périodes de fécondité des femmes (tableau 1). Examinons les comportements des générations, pour les dix dernières années connues dans ces trois intervalles.

NOMIBRI: D'ENFANTS NÉS DIE 100 FEMMES EN FRANCE PAR AN POUR LES TROIS CATÉGORIES D'ÂGE: AVANT 24 ANS, DE 24 À 35 ANS, DE 35 À 50 ANS

\begin{tabular}{|c|c|c|c|c|c|}
\hline Années & $\begin{array}{c}\text { Avant } \\
24 \text { ans }\end{array}$ & à 35 ans & 24 à 35 ans & à 50 ans & 35 à 50 ans \\
\hline 1978 & 78,1 & 216,6 & 138,5 & 264,0 & 47,4 \\
1979 & 76,7 & 213,7 & 137,0 & 262,9 & 49,2 \\
1980 & 75.9 & 209,6 & 133,7 & 263,4 & 53,8 \\
1981 & 74,2 & 204,7 & 130,5 & 261,3 & 56,6 \\
1982 & 72.1 & 200,5 & 128,4 & 261,8 & 61,3 \\
1983 & 68,6 & 198,1 & 129,5 & 260,4 & 62,3 \\
1984 & 66,4 & 196,5 & 130,1 & 259,0 & 62,5 \\
1985 & 62,8 & 196,2 & 133,4 & 257,6 & 61,4 \\
1986 & 60,2 & 195,4 & 135,2 & 253,5 & 58,1 \\
1987 & 56,6 & 195,8 & 139,2 & 251,9 & 56,1 \\
1988 & 52.9 & 195,0 & 142,1 & 247,7 & 52,7 \\
1989 & 49,9 & 195,3 & 145,4 & 243,7 & 48,4 \\
\hline
\end{tabular}

a) Les femmes nées de 1954 à 1965 ont atteint 24 ans entre 1978 et 1989. Le nombre d'enfants qu'elles ont mis au monde dans cet intervalle baisse de 78,1 à 49,9 enfants par an pour $100 \mathrm{femmes,} \mathrm{d'une} \mathrm{façon} \mathrm{remarquablement} \mathrm{régulière,}$ avec une tendance à l'accélération de la vitesse de descente.

b) Les femmes nées de 1943 à 1954 ont atteint 35 ans au cours de cette période. Entre 24 et 35 ans, le nombre d'enfants qu'elles ont mis au monde baisse d'abord de 138,5 (génération 1943) à 128,4 (génération 1947) enfants/an pour 100 femmes, pour remonter ensuite jusqu'à dépasser le seuil initial en atteignant 145,4 dans la génération 1954. Ceci peut s'expliquer :

- par la faible proportion de couples sans enfant en France, couples qui se marient de plus en plus tard,

- par le nombre de plus en plus élevé de jeunes femmes qui entreprennent des études supérieures, ce qui retarde leur âge d'entrée dans la vie active,

- par un retard de l'âge de la fécondité, en notant que l'effet de récupération de ce retard s'est manifesté assez tardivement,

- enfin, par le fait que la baisse de fécondité concerne les rangs de naissance élevés (femmes ayant trois enfants et plus).

c) Les femmes nées entre 1928 et 1939 ont atteint 50 ans au cours de cette période. Le nombre d'enfants qu'elles ont mis au monde entre 36 et 50 ans, qui a augmenté de 47,4 (génération 1929) à 61,5 (génération 1934) enfants/an pour 100 femmes, redescend depuis (48,4 dans la génération 1939).

Ces différentes informations, et notamment a) et b) qui sont plus fraîches que la courbe de descendance finale évoquée au début de notre étude, ne permettent pas d'exclure la simple extrapolation faite ci-dessus. En effet la hausse de la fécondité des générations nées de 1943 à 1954 entre 25 et 35 ans, même si elle perdurait pendant les générations futures, ne compense pas pour l'instant la baisse continue enregistrée par les générations nées de 1954 à 1965 pendant leur période 
de fertilité 14-24 ans, à laquelle s'ajoute maintenant la baisse des naissances aux âges élevés constatée depuis 1984.

Tout cela ne nous donne cependant aucune certitude pour l'avenir. L'évolution future de la fécondité ne peut certainement pas être appréhendée à partir de l'indice synthétique de fécondité, très sensible à la conjoncture et aux décisions prises par le pouvoir pour favoriser ou non la natalité. La courbe de la descendance finale est une donnée structurelle plus régulière, car elle s'appuie sur le cumul des faits constatés au cours des trente-cing années précédentes, sans qu'on puisse cependant établir de lien mathématique entre elle et les observations du moment faites dans le passé. Il sera, d'un point de vue scientifique, intéressant de surveiller l'évolution de la courbe calculce pour la présente note afin de savoir si elle se confirme ou si elle s'infirme et, dans ce cas, dans quel sens.

Gérard-François DUMONT Pierre DESCROIX

\section{JOMARD ET LA DÉMOGRAPHIE DE L'ÉGYPTE}

Pendant son expédition en Égypte (1798-1801), Napoléon était accompagné d'un groupe de savants dans presque toutes les branches du savoir, culture, sociologie, agriculture, ressources, histoire, géographie, etc. A leur retour en France, ces savants passèrent les 17 années suivantes à étudier les observations recueillies, analyser leur matériel et préparer des rapports qui furent publiés sous forme encyclopédique dans la «Description de l'Égypte» à partir de 1809. Une seconde édition, comprenant 26 volumes de texte et 11 d'annexes, fut publiée entre 1821 et 1829 .

Témoignage de la qualité scientifique de ces travaux, un article d'E. Jomard, Commissaire du Gouvernement et coordonateur du groupe, explore divers aspects de la démographie de l'Égypte, malgré l'absence de recensements ou de registres de population, en s'appuyant sur une information très limitée tirée d'autres sources. L'article est intitulé «Mémoire sur la population comparée de l'Égypte ancienne et moderne»; il figure aux pages 103 à 211 du volume IX de la seconde édition. Au-delà de l'hommage rendu à Jomard pour son travail, nous nous proposons d'utiliser cclui-ci pour étendre, par une analyse critique, notre connaissance de la démographie égyptienne moderne.

Bien que le document date de presque deux siècles, Jomard y démontre une bonne maîtrise des aspects fondamentaux de la démographie. Ainsi, il débute en écrivant que «la connaissance de la population d'un pays est non seulement une donnée dont nous avons besoin pour juger de ses ressources, de sa prospérité, en un mot de son existence politique, mais c'est encore un des premiers éléments de l'administration même de l'État, et sans lequel il est presque impossible au gouvernement de comparer la production à la consommation, l'impôt au revenu enfin de régler sûrement l'économie publique». Même pour nous aujourd'hui, cette affirmation constitue une bonne présentation des buts de la statistique démographique.

Jomard note que les Égyptiens anciens avaient compris l'intérêt de bien connaître la population : ils tenaient soigneusement à jour des registres publics et dénombraient précisément l'ensemble des habitants. Les grands monuments qui ont immortalisé le nom du pays en sont une illustration convaincante. La malheureuse 